\title{
Management of Orthopaedic Accidental Emergencies Amidst COVID-19 Pandemic: Our Experience in Preparing to Live with Corona
}

\author{
Vineet Dabas ${ }^{1} \mathbb{D} \cdot$ Nishant Bhatia ${ }^{1} \cdot$ Akash Goel $^{1} \cdot$ Vedpal Yadav $^{1} \cdot$ Vineet Bajaj $^{1} \cdot$ Vinod Kumar $^{1}$
}

Received: 4 August 2020 / Accepted: 2 September 2020 / Published online: 10 September 2020

(c) Indian Orthopaedics Association 2020

\begin{abstract}
Introduction With increasing prevalence of coronavirus cases (including among health care providers), the current advice for orthopaedic surgeons is to favor non-operative management of most injuries and reduce face-to-face follow-up. We present our experience in managing the patients at Government-run non-COVID-19 trauma center in Delhi in an algorithmic form. Our standard operating protocols were mainly based on recommendations of Indian Orthopaedic Association and targeted to provide healthcare at a minimum risk to the treating team as well as other patients admitted to the hospital.

Methodology We describe the inflow, in-hospital management and outflow of patients at our facility during the lockdown period and in the following unlock period (from 23 March to 8 July 2020). Those patients who had absolute indications for surgery were offered surgery, while conservative treatment was more favored in those with relative indications. We also highlight the changes incorporated in OT settings as well as in rehabilitative and follow-up period.

Results Following the described protocol helped us maintain a balance between the safety of patients and our front line workers which was evident by very low COVID-19-positive rate in admitted patients (4.22\%) and health care providers $(16.67 \%)$ in the above-mentioned time period.

Conclusions We need to be prepared to cohabitate with this deadly Novel Coronavirus and adapt our surgical practices according to the need of the hour by minimizing surgical indications and strengthening the training in conservative principles.
\end{abstract}

Keywords Coronavirus $\cdot$ Orthopaedic emergencies $\cdot$ Pandemic $\cdot$ Trauma $\cdot$ Healthcare provider $\cdot$ Protection

\section{Introduction}

Vineet Dabas

vineetdabas@yahoo.com

Nishant Bhatia

bhatianishant1111@gmail.com

Akash Goel

akashgoyal1993.cool@gmail.com

Vedpal Yadav

vyadav2002@gmail.com

Vineet Bajaj

vineet7bajaj@gmail.com

Vinod Kumar

drvk706@gmail.com

1 Department of Orthopaedics, Maulana Azad Medical College and Associated Lok Nayak Hospital, New Delhi, India
The first reports of coronavirus (COVID-19) outbreak came from Wuhan city of China in the month of December 2019 [1]. It was declared as a global pandemic by World Health Organization (WHO) on 11 March 2020 [2]. As of 25 July $2020,15,296,926$ cases and 628,903 deaths have been reported worldwide [3]. In India, the first case of COVID19 was reported on 30 January 2020, and the number has reached $1,287,945$ as on 25 July 2020 , with 30,601 deaths [4]. The most effective strategies against COVID-19 include preventing exposure and staying indoors. Keeping this in mind, the Government of India (GOI) declared a nationwide lockdown on 23 March 2020 for a period of 3 weeks which was extended further to 3rd May. The lockdown was still further extended till 17th May but this time with ease of some restrictions. Thereafter, unlock procedure was started in many states and over the time period, several states have reopened various public facilities with adequate precautions. The GOI has designated the 733 districts of India into 
red, orange and green zones according to varying levels of restriction aimed at containing the virus [5]. Restrictions are being imposed/withdrawn depending upon the rate of increase/decrease in number of active cases across various states [6]. We are still far away from the pandemic being over and now is the time that we start implementing our exit strategies and prepare ourselves to coexist with this deadly disease.

With increasing prevalence of cases in Delhi hospitals with affected healthcare workers, the current advice is to take pragmatic decisions and favor non-operative management of most injuries and reduce face-to-face follow-up [7]. In orthopedic surgical procedures, power tools, such as electrocautery, bone saws, reamers and drills, are commonly used that have shown to produce aerosols [8]. It is now well documented that virus transmission can happen through blood aerosols infecting the health care professionals [9].

We present our experience in managing patients with acute trauma presenting to the Government-run trauma center in Delhi. Keeping in mind the future challenges in the post-lockdown period when a sudden surge of orthopaedic emergencies is expected again, we highlight the algorithmic approach adopted by our institute and expect it to be useful in the coming time when uncertainty regarding the end of this pandemic will still persist and we will be expected to coexist with the virus around.

\section{Methods}

The study collected data from the period 23 March to 8 July 2020 at Government-run trauma center in Delhi. The standard operating procedures (SOPs) were implemented which were based upon the recommendations of ICMR, Ministry of Health and Family Welfare, GOI and Indian Orthopaedic Association (IOA) [10], and targeted to provide optimum healthcare at a minimum risk to the treating team as well as other patients admitted to the hospital.

\section{Infrastructure Preparedness}

Sanitization of the Facility Spraying of the surfaces and mopping of the floors were done with $1 \%$ hypochlorite solution every $12 \mathrm{~h}$ in areas including wards, ICU, offices, gas plant, doctors' duty rooms, entrances and exits, etc. For the emergency ward where patients were received, deep cleaning and sanitization was done every $6 \mathrm{~h}$ and on SOS basis (in cases of spills, etc.). Fomites and workstation cleaning were carried out every $3-4 \mathrm{~h}$ with $0.5-1 \%$ hypochlorite. Strict measures were taken to avoid any unnecessary fomites like papers, forms, boxes, etc. on counters.

Receiving the Patients in Emergency Separate corridors for entry and exit of patients and health care workers (HCW) were designated so as to avoid risking the work force which is probably the most important resource needed at present times. The emergency wards were designated into isolation and non-isolation zones. Whenever possible, all HCWs in the emergency were advised to wear full PPE (Personal Protective Equipment), kits (hazmat suits and face shields included) while receiving, examining and resuscitating patients. In these times of crisis, shortage of adequate equipment is not an unexpected event. Hence, the minimum protective items that were made absolutely necessary before receiving any patient were N95 mask, face shield, head cap, examination gloves, shoe covers and a surgical gown. All the patients were screened with an infrared thermometer and history pertaining to COVID-19 symptoms like fever, dry cough, difficulty in breathing, contact with a known positive case, travel, etc. was elicited. If there was any positive history, the patient was sent to isolation zone and was assumed to be a suspect, while patients with no history of COVID-19 like symptoms were shifted to non-isolation zones. CT scans were ordered only when deemed absolutely necessary like in cervicodorsal spine. The imaging rooms were sanitized frequently as per the recommendations of CDDC [11].

\section{Primary Treatment}

Among the patients shifted to isolation zone (COVID-19 suspects), those requiring non-operative treatment were managed with fluids, analgesics, closed reduction and splintage in plasters, etc. as required for the particular injury. The threshold for the need of operative intervention was kept very high in such patients. These patients were discharged at the earliest and referred to dedicated COVID-19 hospitals for further testing and management of their COVID-19-like symptoms. Suspected COVID-19 patients who were stable but had a clear-cut need for surgery, like femoral neck fractures, closed displaced intra-articular fractures, irreducible long bone fractures, etc. were given primary treatment in the form of fluids, temporary stabilization with splints, plasters and dressings. They were referred to dedicated COVID-19 centers for surgical management in ambulances with $\mathrm{HCW}$ in PPE and maintaining special precautions like social distancing, coughing and sneezing etiquettes.

Patients who were hemodynamically unstable or required a limb/life-saving surgery (whether COVID-19 suspect or not), for instance, grade three open fractures, vascular injury, compartment syndrome, mangled extremity, pelvic injuries requiring fixation, implant-related sepsis (in those who were previously treated at our facility) were considered to be COVID-19-positive and shifted to isolation zone. They were managed with full COVID-19 precautions as discussed further.

Among the non-COVID-19 suspects, who were shifted to non-isolation zone, those who could be managed 
conservatively were given adequate primary treatment like fluids, analgesics and splintage and were discharged at the earliest so as to minimize the risk of infection transmission to them as well as health care workers. All attempts were made to manage the patients non-operatively. Those patients who had absolute indications for surgery were offered surgery, while the relative indications for surgery in a particular injury were considered as low priority. This policy was used as our center was one of the few non-COVID-19 centers having the facility to perform fracture fixations requiring $\mathrm{C}$-arm, and thus there was a need to give preference to the absolute indications. This usefulness of this policy became more evident once the lockdown started lifting and patients coming to our center increased.

The remaining patients were broadly divided into three categories:

1. Patients needing non-operative treatment.

2. Patients needing operative treatment but a delay of a couple of days do not adversely affect the outcome for the patient.

3. Patients requiring urgent surgery where a delay could adversely affect the outcome.

For the first two categories of patients, all the necessary preoperative investigations and their COVID-19 samples (throat and nasopharyngeal swab RT-PCR) were sent at the earliest. The surgeries were performed after receiving the COVID-19 reports. This also gave time to observe the patients at hospital for any signs of COVID-19 infection. The patients with injuries like proximal femur fractures, intra-articular fractures and unstable spine injuries with partial neurological deficit were given priority in the OT list. Overall, patients, in whom conservative management or delayed primary surgery would hamper earning of livelihood, were considered for surgical management.

For the third category of patients, they were considered COVID-19-positive, and were operated taking all the precautions as per the guidelines for Personnel protection. The RT-PCR test for COVID-19 was sent the very next morning for these patients. This was done so that if the patient comes to be positive for COVID-19, the team involved in the surgery could be quarantined as their exposure to aerosols would be high. Once the Rapid test for COVID-19 became available to us, all these patients were tested using the Rapid test kit before shifting them to OT.

Segregation in the Wards The preoperative wards were divided into 'COVID Report Awaited' and 'COVID Report Negative' rooms. Adequate distancing was maintained between beds and full measures were taken to maintain social distancing among attendants as well as health care workers. Proper measures were taken to ensure hand hygiene, use of masks by patients and their attendants, avoiding social gatherings at times of eating, coughing and sneezing etiquettes, etc. All patients and their attendants were required to wear triple-layered surgical masks.

Patients who tested positive for SARS-COV 2 were transferred to dedicated COVID-19 facility, to which our center is affiliated. Proper measures were taken to ensure the safety of the accompanying healthcare worker to avoid transmission. Those who tested negative were shifted to 'COVID Report Negative' rooms and prepped up for surgery as soon as possible. Figure 1 is a flow chart depicting the SOP adopted by our facility during the COVID-19 pandemic.

\section{Operative Management}

Theater Settings One operating room out of the two available to us was dedicated for patients needing emergency surgery (where COVID-19 status was unknown). Even when the testing was available, everyone was alert to the possibility of the test being false negative and precautions were carried out even in these patients. Proper donning and doffing areas were designated for equipping PPE kits. Anesthetic induction and use of orthopaedic power tools are aerosol generating procedures and require negative pressure maintenance in OTs [12]. Minimum number of personnel were allowed inside the OT at any given time. All theater staff were advised to wear enhanced PPE that consists of FFP3mask (Filtering face piece and the number denotes level of protection), full face shield, head cover, double pair of gloves, full sleeve waterproof gown and shoes. All staff were trained to do donning and doffing in the designated areas provided. General Anesthesia (GA) involving intubation has shown to increase the production of aerosols leading to an increased risk of infection to the healthcare workers [9]. The aim was to do most of the procedures under regional block, but this was not possible for all procedures. If GA was administered, then it was carried out with minimal number of staff. The same was applied when the patient was extubated [13]. Recovery of the patient was done in theater, and once stable, he/she was shifted directly to post-operative ward. Another important aspect in OT setting was careful management of waste. All the wastes pertaining to COVID-19 patients (suspects/positives) were discarded in dedicated yellow bags, be it plastic/ cotton/latex or human waste.

Changes in Surgical Practices Cautery use was minimized. An assistant was handed the task to constantly operate the suction machine. Syringe wash was used along with suction instead of direct and pulsed lavage. Operating time and blood loss were tried to be kept minimal. Objective was to achieve quick and adequate fixation. Hammering of implants was carried out only after covering the field with absorbent linen. Whenever it was possible, a senior and experienced surgeon would perform most cases so as to reduce the operating time, blood loss and execute quick 
Fig. 1 Standard operating protocol at trauma center

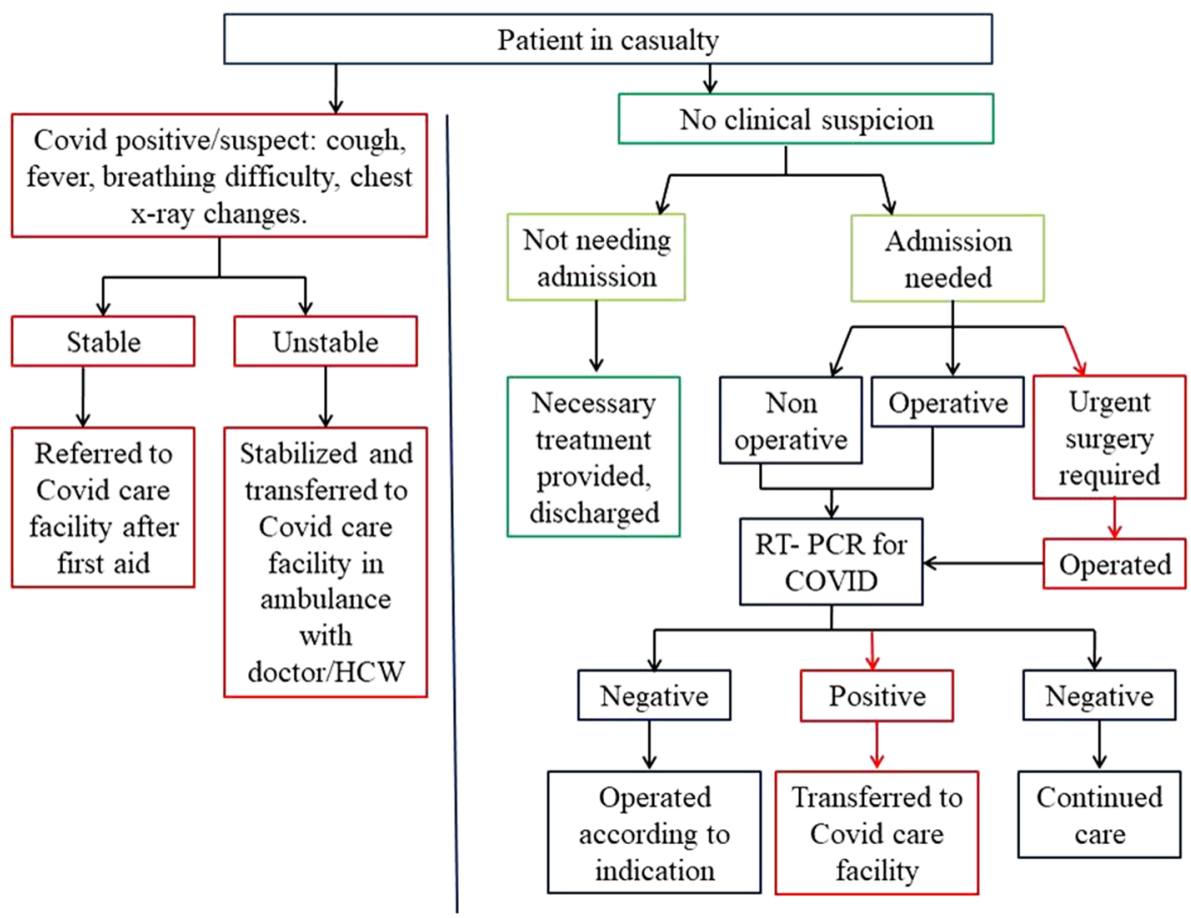

exposure and fixation. Drains were avoided. Every precaution was taken to prevent any kind of spillage.

\section{Postoperative Care, Follow-up and Rehabilitation}

The post-operative stay of the patient was tried to be kept to minimum required. After observing the patients for 1-2 days, they were discharged mostly on oral analgesics and antibiotics; however, if intravenous drugs/frequent dressings, etc. were needed, duration of stay was extended. Follow-up visits were restricted to suture removal days. A separate floor was designated to run the follow-up OPD. Most of the complaints and apprehensions of the patients were attended telephonically. During the postoperative stay, the patients were told about what exercises they needed to do and at what time in sessions conducted by our physiotherapist with adequate social distancing. The patients who were COVID-19-positive (including those who tested positive after emergency surgery) were shifted to our COVID19-dedicated facility for the further management of their illness after they were deemed stable for transportation. Stay of such patients was tried to be kept as small as possible.

\section{Healthcare Providers}

To carry out this protocol effectively, while keeping the exposure of the healthcare workers to minimum, we divided our team of doctors into two units. Each unit worked at a time in divided shifts for 15 days followed by a quarantine period of 15 days. The other unit joined after the 1 st unit had finished 15 days of its work. This division of the work force into units was done on the premise that if even one patient with COVID-19 infection was operated or admitted in the ward for a significant duration, the team coming in contact would need to be quarantined else they would place the nonexposed staff at risk. With two teams, it would be possible to replace the quarantined team.

\section{Results}

A total of 794 patients presented to the center who were managed as in-patient and out-patient as shown in Table 1. Five $(3.5 \%)$ out of 142 admitted patients were tested positive in the pre-operative period and only one $(0.7 \%)$ patient was positive in the post-operative period.

Table 1 Distribution of patients according to treatment strategies $(N=794)$

Admitted $(n=142)$ Discharged $(n=652)$

Major surgery- 85 (10.71\%)

Minor surgery- $36(4.54 \%)$

Needing surgery but managed conservatively- 377 (47.48\%)

Significant injuries but not needing surgery- 275 $(34.64 \%)$

Conservative- 21 (2.64\%) 
Fifteen $(1.89 \%)$ patients with open fractures required emergency surgery before COVID-19 sampling could be done. One patient with fracture of lumbar spine with partial neurological deficit (including bladder and bowel involvement) was planned for early decompression and stabilization of the spine, who was detected as COVID-19-positive later. All the members of the operative team (including the anesthetist and the paramedical staff) were quarantined for a week in the post-op period and were tested negative after 7 days.

Table 2 shows the COVID-19 positivity rate among HCWs with an overall rate of $16.67 \%$, the source of infection, however, could not be validated in them. Out of total 17 COVID-19-positive individuals, seven (41.2\%) were asymptomatic, 10 (58.8\%) were mildly symptomatic, and none were seriously ill requiring intensive care. Most of these infections were during the first week of lockdown and more than $50 \%$ of the workers were of the same category, sharing a common room. This practice of sharing room by multiple workers was discontinued after the initial spike.

\section{Discussion}

The present study discusses the management principles of orthopedic trauma during COVID-19 pandemic in Delhi. Patients having emergent conditions like grade three open fractures, mangled extremities, vascular injury, polytrauma or compartment syndrome need to be considered as COVID19-positive (or suspects) and should be managed on urgent basis (with enhanced PPEs) with appropriate resuscitation and operative intervention. Enhanced PPEs and special considerations like separate isolated wards, on table intubation and extubation, minimal use of orthopaedic power tools, reducing the operating time and blood loss, aiming for quick and adequate fixation instead of aggressive and rigid fixation are some extremely important measures that we need to adopt in these times of crisis when the infection is rampant in our health care force leading to compromised patient care and increased stress on our already overburdened medical system. So far in India and other South Asian countries, we do not have specific and detailed guidelines for management

Table 2 Testing in health care providers (RT- PCR through nasopharyngeal/oropharyngeal swab)

\begin{tabular}{lcc}
\hline & Working on duty & Tested positive \\
\hline Doctors & 33 & $2(9.5 \%)$ \\
Physiotherapist & 1 & $1(100 \%)$ \\
Nurses & 30 & $3(10 \%)$ \\
Other workers & 38 & $11(29 \%)$ \\
Total & 102 & $17(16.67 \%)$ \\
\hline
\end{tabular}

of orthopaedic accidental injuries. The IOA and BOA have published some extensive guidelines; however, the experience and consensus regarding those are remotely available. Hence, the experience we are sharing here may be of value in enhancing the acceptability of such guidelines especially in the burdened Government set-up with minimal facilities and when there was no clarity about resolution of this pandemic. We were able to operate the negative patients within $48 \mathrm{~h}$ of their test reporting. During the surgery of these negative patients, we did not believe it was absolutely necessary to wear full PPE although the choice was left to individuals getting scrubbed in the surgery. Spending long duration in full PPE hampers the proficiency of the chief operating surgeon but operating the patient within $48 \mathrm{~h}$ of negative report without hazmat suits (which are not available at most places) definitely allowed us to pull off the best possible surgical outcome.

Operative management of fractures provides early rehabilitation and highest level of function to the patient with minimal residual disability; however, during this pandemic and in the near future, we have to be very careful in such an approach as the patients with COVID-19 infection (which may be undetected) are known to have higher complication rates when operated under anesthesia. Hence, there is an urgent need to shift back to conservative principles of fracture management and keep the threshold for operative intervention at a higher level. This will ensure the safety of the frontline task force, as was evident from our experience and control the cross-spread of infection to patients coming to the hospital. These changes need to be adopted till efficacious and safe vaccine and targeted drugs for treatment and prevention become available in market.

Recently, Rapid Antigen Test (RAT) from nasopharyngeal swab has been made widely available at all Government facilities. Although the test is useful in screening the patients coming to the casualty, keeping in mind the massive exposure while performing a surgery, we still recommend an RT-PCR test before taking up a patient for surgery as the sensitivity of rapid test is low (30-45\%) [14-16].

From the point of view of the infrastructure, the lack of single isolation rooms in our facility was one of the main hurdles. Initially, during the lockdown, the admissions were less and we could keep the patients on alternate beds. But this could not be maintained once the admission rate increased. Another issue was the availability of a single OT complex, making it impossible to maintain a separate facility for COVID-19-positive/status unknown patients.

Proper planning and execution of policies to de-stress our health system and improve patient care without risking the health of its frontline task force is one long mile that this nation needs to cover to survive this and any future pandemics. We believe that we were able to manage our facility well with whatever limited resources we had by following 
this approach. Further changes need to be adopted as and when new technological advancements are made pertaining to the disease.

\section{Conclusion}

The most important lesson for orthopaedic surgeons is that effective and necessary treatment of patients should be provided while ensuring safety of the health care workers during this pandemic. We need to be prepared to cohabitate with this deadly Novel Coronavirus and adapt our surgical practices according to the need of the hour. Minimizing surgical indications, strengthening the training in conservative principles and following protocols indigenized for workplaces can provide optimum patient care with personal safety.

Authors' Contributions VD: conceptualization, methodology, validation, supervision, writing- original draft, writing- review and editing. NB: methodology, data curation, writing- original draft. AG: data curation, writing- review and editing. VY: conceptualization, methodology, writing- review and editing. VB: conceptualization, methodology, writing- review and editing. VK: validation, supervision, writing- review and editing.

Funding No funds were received in support of this work.

Data Availability Data generated during and/or analyzed during the current study are not publicly available due to confidentiality reasons but are available from the corresponding author on reasonable request.

\section{Compliance with Ethical Standards}

Conflict of interest The authors declare that they have no competing interests.

Ethical standard statement This article does not contain any studies with human or animal subjects performed by the any of the authors.

Informed consent For this type of study informed consent is not required.

\section{References}

1. Azman, A. S., \& Luquero, F. J. (2019). Comment from China: hope and lessons for COVID-19 control. The Lancet Infectious Diseases, 20, 2019-2020.

2. Vannabouathong, C., Devji, T., Ekhtiari, S., Chang, Y., Phillips, S. A., Zhu, M., et al. (2020). Novel coronavirus COVID-19: current evidence and evolving strategies. The Journal of Bone and Joint Surgery, 102, 734-744.

3. World Health Organisation. Coronavirus disease (COVID-19): Situation report 186. https://www.who.int/docs/default-source/ coronaviruse/situation-reports/20200724-COVID-19-sitrep-186. pdf?sfvrsn=4da7b586_2. Accessed 25July 2020.

4. Government of India. COVID-19. https://www.mygov.in/COVID -19. Accessed 25 July 2020.

5. India Today. Full list of red, yellow, green zone districts for lockdown 3.0. https://www.indiatoday.in/india/story/red-orange-green -zones-full-current-update-list-districts-states-india-coronaviru s-1673358-2020-05-01. Accessed 10 May 2020.

6. India Today. Coronavirus: Ghaziabad DM extends lockdown restrictions till May 31. https://www.indiatoday.in/india/story/ coronavirus-up-ghaziabad-lockdown-extended-may-31-16747 33-2020-05-05. Accessed 10 May 2020.

7. British Orthopaedic Association. Management of Patients with Urgent Orthopaedic Conditions and Trauma during the Coronavirus Pandemic. London: British Orthopaedic Association; 2020. https://www.boa.ac.uk/resources/COVID-19-boasts-combined. html. Accessed 10 May 2020.

8. Yeh, H. C., Turner, R. S., Jones, R. K., Muggenburg, B. A., Lundgren, D. L., \& Smith, J. P. (1995). Characterization of aerosols produced during surgical procedures in hospitals. Aerosol Science and Technology, 22, 151-161.

9. Tran, K., Cimon, K., Severn, M., Pessoa-Silva, C. L., \& Conly, J. (2012). Aerosol generating procedures and risk of transmission of acute respiratory infections to healthcare workers: a systematic review. PLoS ONE, 7, e35797.

10. Indian Orthopaedic Association. COVID-19 IOA Guidelines. New Delhi: Indian Orthopaedic Association; 2020. https://www.ioain dia.org/COVID19IOAguidelines.pdf. Accessed 10 May 2020.

11. CDC. Environmental cleaning. https://www.cdc.gov/hai/pdfs/ resource-limited/environmentalcleaning. Accessed 10 May 2020.

12. Raghavan, R., Middleton, P., \& Mehdi, A. (2020). Minimising aerosol generation during orthopaedic surgical procedures- current practice to protect theatre staff during COVID-19 pandemic. J Clin Orthop Trauma. https://doi.org/10.1016/j.jcot.2020.04.024.

13. COVID-19: infection prevention and control (IPC). Public health England. 10 January 2020. Last updated 12 April 2020.

14. Scohy, A., Anantharajah, A., Bodéus, M., Kabamba-Mukadi, B., Verroken, A., \& Rodriguez-Villalobos, H. (2020). Low performance of rapid antigen detection test as frontline testing for COVID-19 diagnosis. Journal of Clinical Virology, 21, 104455.

15. Döhla, M., Boesecke, C., Schulte, B., Diegmann, C., Sib, E., Richter, E., et al. (2020). Rapid point-of-care testing for SARS-CoV-2 in a community screening setting shows low sensitivity. Public Health, 182, 170-172.

16. Mak, G. C., Cheng, P. K., Lau, S. S., Wong, K. K., Lau, C. S., Lam, E. T., et al. (2020). Evaluation of rapid antigen test for detection of SARS-CoV-2 virus. Journal of Clinical Virology, 8, 104500. https://doi.org/10.1016/j.jcv.2020.104500.

Publisher's Note Springer Nature remains neutral with regard to jurisdictional claims in published maps and institutional affiliations. 